\title{
NIVELES DE LAI/fPAR EN SUPERFICIES AFECTADAS POR INCENDIOS FORESTALES EN ARAGÓN. ANÁLISIS MEDIANTE EL PRODUCTO MCD15A2 DE MODIS
}

\section{LAI/fPAR levels on surfaces affected by forest fires in Aragón. A MODIS MCD15A2 analysis}

\author{
Adrián Jiménez Ruano, Fernando Pérez-Cabello* y Raquel Montorio Llovería \\ Departamento de Geografía y Ordenación del Territorio, \\ Instituto de Investigación en Ciencias Ambientales (IUCA), \\ Universidad de Zaragoza, C/ Pedro Cerbuna, 12, 50009. Zaragoza. \\ *Autor corresponsal: fcabello@unizar.es
}

Recibido: 24-03-2015. Aceptado: 05-04-2016. Fecha de publicación on-line: 07-06-2016

Citation / Cómo citar este artículo: Adrián Jiménez Ruano, Fernando Pérez-Cabello y Raquel Montorio Llovería (2016). Niveles de LAI/fPAR en superficies afectadas por incendios forestales en Aragón. Análisis mediante el producto MCD15A2 DE MODIS. Pirineos, 171, e019. doi: http://dx.doi.org/10.3989/Pirineos.2016.171003

\begin{abstract}
RESUMEN: El índice de área foliar (LAI) y la fracción de radiación activa fotosintética absorbida por la vegetación (fPAR) son variables relacionadas con la estructura del dosel vegetal que pueden aportar nuevas claves en la comprensión del proceso de regeneración vegetal en zonas quemadas. En este contexto, el objetivo del trabajo es caracterizar diferentes superficies quemadas en Aragón en función del producto MDC15A2 (LAI/fPAR) de MODIS. Se realizan dos tipos diferentes de análisis: (1) análisis estático de los valores de LAI/fPAR a partir del compuesto de 2010; (2) seguimiento multianual en 6 grandes incendios ocurridos en la década de los 2000, representativos de condiciones ambientales diferentes. El proceso metodológico se basa en la selección de 18 incendios forestales $(>500$ ha, ocurridos entre 1975-2010) que dispusieran de la cartografía digital; y en la descarga del producto MCD13A2 de MODIS (compuestos estacionales de 8 días, primera semana de mayo y septiembre).

Se ha identificado un patrón temporal de recuperación en los valores de LAI. En incendios ocurridos hace más de 35 años se recogen valores promedio de LAI $>1$ (1,13/1,40, en septiembre y mayo respectivamente), y valores de fPAR bajos $(\sim 0,5)$. Mientras, un año después del fuego los valores promedio de LAI/fPAR no superan el 0,20/0,40, respectivamente. Por otro lado, unos meses tras el fuego se han registrado valores promedio de $\mathrm{LAI}<0,25$. En términos generales, el producto MODIS MDC15A2 (LAI/fPAR) presenta un enorme potencial en el análisis cartográfico de los procesos biológicos de zonas quemadas, a pesar de los problemas de interpretación derivados de la resolución espacial del producto.
\end{abstract}

PALABRAS CLAVE: Incendio forestal; LAI; fPAR; MODIS; Aragón.

ABSTRACT: The Leaf area index (LAI) and the Fractional Photosynthetically Active Radiation (fPAR) are variables related to the structure of the plant canopy that can provide new keys for the understanding of post-fire vegetation processes. In this context, the objective of this work is to characterize different burned areas in Aragon using the MDC15A2 (LAI/fPAR) product from MODIS. There are two different types of analysis: (1) static analysis of LAI/ fPAR values from the compound of 2010; (2) multi-year follow-up in 6 large fires occurred in the 2000s, representa- 
tive of different environmental conditions. The methodological process is based on the selection of 18 forest fires (> 500 ha, occurred between 1975-2010) with digital cartography available; and the download of the product MCD13A2 MODIS (seasonal compounds of 8 days, first week of May and September).

A temporal pattern of recovery in the values of LAI has been identified. Fires occurred more than 35 years ago have average LAI values $>1(1.13 / 1.40$, in September and May respectively), and low values of fPAR $(\sim 0.5)$. Meanwhile, a year after the fire LAI/fPAR average values do not exceed $0.20 / 0.40$, respectively. On the other hand, a few months after the fire average LAI values $<0.25$ have been observed. In general terms, the MODIS MDC15A2 (LAI/ fPAR) product features an enormous potential in the cartographic analysis of the biological processes of burned areas, despite the problems of interpretation derived from the spatial resolution of the product.

KEYWORDS: Wildfire; LAI; fPAR; MODIS; Aragón.

\section{Introducción}

En el contexto de los ecosistemas mediterráneos, los incendios forestales se han convertido en las últimas décadas en uno de los problemas ambientales de mayor significación, como consecuencia de su alta ocurrencia y magnitud en términos de superficie afectada.

Aunque la acción del fuego afecta al sistema suelo-vegetación-fauna, la destrucción de la vegetación mediante quema directa y/o deshidratación de los tejidos constituye el efecto más inmediato y de mayor envergadura ecológico-paisajística. Sin embargo, después de un incendio, en ecosistemas adaptados al fuego, se produce una intensa profusión de los mecanismos de rebrotación/germinación de las especies preexistentes e invasivas, que en gran medida determinan el ritmo e intensidad del proceso de regeneración vegetal (Mooney \& Hobbs, 1986; Pausas, 2004).

En este marco, un primer paso a la hora de comprender los efectos y la dinámica ambiental tras el fuego lo constituye la monitorización de la respuesta de la vegetación afectada, por lo que todavía hoy sigue siendo necesario establecer métodos de diagnóstico de los efectos del fuego y protocolos de seguimiento en zonas quemadas.

A nivel de parcela en la literatura podemos encontrar numerosas experiencias en donde el seguimiento de la regeneración postincendio se realiza mediante la identificación de una serie de parámetros, medibles en campo, como los niveles de recubrimiento vegetal o la composición florística. En cambio, no es tan frecuente la medición de parámetros biofísicos tales como el índice de área foliar -LAI (Leaf Area Index)- o la Fracción de Radiación Activa Fotosintética -fPAR (fraction of Absorbed Photosynthetically Active Radiation); parámetros que podrían ser utilizados como indicadores del grado de afección y de la recuperación del funcionamiento eco-fisiológico de las zonas quemadas.

El LAI es una propiedad estructural del dosel que hace referencia a la cantidad de vegetación distribuida en la vertical, definiéndose como la superficie foliar fotosintéticamente activa (contabilizada por una sola cara) por unidad de suelo (Chen \& Black, 1992). Por su parte, la Fracción de Radiación Activa Fotosintética mide la fracción de la radiación incidente sobre una bóveda foliar (entre los 400 y 700 nanómetros) que es absorbida por las hojas para transformarla en energía química durante la fotosíntesis.
No obstante, indistintamente de que se utilicen parámetros fisionómicos, florísticos o biofísicos, el carácter puntual de los datos procedentes de parcelas de campo arrastra el problema del grado de representatividad para el conjunto de la superficie afectada. En este sentido, los datos de teledetección espacial se han erigido como una solución, gracias a que proporcionan información espectral sensible a los fenómenos que se producen en áreas afectadas por el fuego con una resolución temporal suficientemente detallada (Chuvieco et al., 2008).

Las imágenes de satélite LANDSAT y, en concreto, el NDVI (Normalized Difference Vegetation Index) han sido hasta la fecha uno de los tándems más empleados (Viedma et al., 1997; Díaz-Delgado \& Pons, 2001; Pérez-Cabello, 2012; Vicente-Serrano et al., 2011). Sin embargo, el NDVI presenta algunos problemas relacionados con la influencia del suelo en zonas de escaso recubrimiento vegetal, o con la saturación en zonas con valores de LAI >3 (Huete, 1988; Gao, 1996; Glenn et al., 2008; Gilabert et al., 1997) y requiere la realización de ajustes empíricos multitemporales para obtener información continua de carácter biofísico. Estos modelos de base empírica no siempre son posibles o representativos para amplias superficies, de hecho existen estudios en los que se describen bajas correlaciones entre LAI y el NDVI (Turner et al., 1999; Cohen et al., 2003).

El sensor MODIS (Moderate Resolution Imaging Spectroradiometer), a bordo de las plataformas TERRA y AQUA, lanzadas en 1999 y 2002, respectivamente, es uno de los más importantes en el seguimiento de procesos de cambio sobre la superficie terrestre por sus características espaciales y espectrales (Justice et al., 2002). Además, junto a las imágenes en 36 bandas discretas (desde $\operatorname{los} 0,405$ a $14,5 \mu \mathrm{m})$, MODIS también proporciona diferentes productos enfocados directamente al estudio de la vegetación. Es el caso del producto MCD15A2, que recoge información de dos variables biofísicas (LAI y fPAR) que, tal y como se ha mencionado, pueden aportar nuevas claves en la comprensión del proceso de regeneración vegetal en zonas quemadas.

En concreto, el producto MCD15A2 de MODIS combina información de los satélites TERRA y AQUA en un compuesto de 8 días a una resolución de $1 \mathrm{~km}$. El algoritmo principal de LAI se basa en un modelo 3D de transferencia radiativa (Knyazikhin et al., 1998) que se invierte mediante una LUT (Look Up Table) específica para dife- 
rentes tipos de biomas en los que se clasifica la vegetación a escala global. Además, utilizan otros productos MODIS, como los productos MOD09 y MOD12, junto a fuentes de información auxiliar.

El primer producto (MOD09) se sintetiza tras una fase de procesamiento de datos, donde se combinan dos bandas de reflectividad (1 y 2 del sensor) y los ángulos solares, obteniendo un producto derivado con el código MODAGAGG, que ya recoge el paso de reflectividades a 250 o 500 metros de resolución, a la normalizada y corregida de $1 \mathrm{~km}$.

El segundo (MOD12Q1) se basa en la cartografía de 6 ecosistemas modelos: pastos y cultivos cerealistas, arbustos, cultivos de hoja ancha, sabana, bosques de hoja ancha y bosques de coníferas. El conjunto de datos auxiliares utilizados (MOD15_ANC_RIx.hdf), recoge información del modelo de transferencia radiativa, los patrones de clasificación de las cubiertas, las propiedades ópticas de la hoja y las soluciones a un conjunto de problemas relacionados con la incertidumbre y las soluciones aceptables de los cálculos.

Los resultados de los mencionados productos están validados mediante la realización de comparaciones con datos tomados in situ y productos de otros sensores (Morisette et al., 2002 y 2003). En este misma línea, en la literatura también encontramos numerosos trabajos (Yang et al., 2006; Fensholt et al., 2004; Serbin et al., 2013) en donde se describe cierta sobreestimación de los productos de LAI/fPAR. Más recientemente, Serbin et al. (2013) incluyeron la componente espacio-temporal en el proceso de validación en bosques boreales, concluyendo que dichos productos recogen satisfactoriamente la trayectoria fenológica de estas formaciones arbóreas, aunque de nuevo apuntan los problemas de sobreestimación en formaciones vegetales jóvenes.

En este marco, el objetivo de este trabajo es caracterizar, desde el punto de vista de los valores LAI/fPAR, diferentes superficies afectadas por incendios forestales ocurridos en Aragón, mediante el análisis de los valores extraídos del producto MODIS MCD13A2. A partir de este trabajo se intenta responder a los siguientes interrogantes: (1) ¿Qué niveles de fPAR-LAI presentan zonas que han sido afectadas por incendios forestales a lo largo del periodo (1975-2010) en Aragón? (2) ¿Cuál es el impacto inmediato y la evolución multianual de los valores de LAI en casos concretos situados en diferentes marcos geográficos de Aragón?

\section{Material y métodos}

\subsection{Selección de superficies afectadas por incendios forestales}

El primer paso de la metodología aplicada en este trabajo consistió en la selección de una serie de incendios representativos de las comunidades vegetales afectadas por el fuego en Aragón que, además, reunieran ciertos requisitos en cuanto a sus dimensiones (superficie quemada $>500$ ha) y distribución espacial (ubicación dentro de un único tile de MODIS). Para ello se contó con la base de datos de incendios forestales del Gobierno de Aragón proporcionada por el Servicio de Gestión de los Incendios Forestales y Coordinación (Dirección General de Gestión Forestal) y de las cartografías en formato digital generadas en el contexto del proyecto GA-LC-042/2011 (Caixa-DGA), mediante técnicas de teledetección y fuentes de datos oficiales.

La selección de los incendios se centró en aquellos ocurridos entre 2000 y 2010, con el propósito de realizar un seguimiento anual de los valores de LAI/fPAR y del impacto del fuego en los primeros meses. Además, la selección se completó incluyéndose incendios antiguos, desde 1975, cuya localización coincidiera con el tile 17h y $4 \mathrm{v}$, coordenadas del extracto de los productos MODIS, con el objeto de evaluar el estado actual de los valores LAI/fPAR en incendios ocurridos en diferentes periodos de tiempo.

En total, se seleccionaron 18 perímetros, espacialmente repartidos en las provincias de Zaragoza y Huesca (Aragón) (Tabla 1 y Figura 1). Debido a la resolución del producto MCD15A2 de MODIS $\left(1 \mathrm{~km}^{2}\right)$, se seleccionaron sólo los píxeles incluidos completamente en el perímetro del incendio, eliminándose los de borde o todos aquellos que, aun estando dentro del perímetro de la superficie afectada, presentaran sectores con zonas de vegetación sin afectar.

La Figura 1 recoge la distribución espacial de los incendios forestales analizados. Se trata de incendios que se distribuyen a lo largo de un eje NE-SO en la zona occidental de Aragón, pudiéndose agrupar en 4 grandes marcos geográficos: incendios localizados en la Ibérica zaragozana, incendios del CENAD (Centro Nacional de Adiestramiento "San Gregorio"), incendios de las sierras prepirenaicas, e incendios de Zuera y los Montes de Castejón. Estos marcos de referencia espacial presentan cierta homogeneidad en relación a las formaciones vegetales afectadas, condiciones fisiográficas y dominios climáticos.

Los incendios con los códigos 6, 7, 11, 13, 17, 19, 26, 29 y 31 recogen amplios sectores quemados integrados en las sierras occidentales del Prepirineo oscense y zaragozano, cubiertos por comunidades de carácter submediterráno dominadas por Quercus gr. cerrioides (Willk y Costa) y, en menor medida, por Quercus ilex L.; pinares de Pinus sylvestris L. y Pinus nigra (Arnold) y formaciones arbustivas dominadas por Buxus sempervirens L., Genista scorpius L., etc. (Pérez-Cabello, 2002; Ruiz de la Torre, 1990a). Los incendios con los códigos 10 y 34 recogen información de los incendios de los Montes de Zuera y Castejón de Valdejasa, siendo representativos de formaciones arbóreas dominadas por Pinus halepensis L., acompañadas por $Q$. coccifera $\mathrm{L}$. y otras especies típicas de matorral mediterráneo. Los incendios con códigos 32 y 38 se localizan en el CENAD y remiten a formaciones vegetales en su mayoría de carácter subarbustivo y pastizales xerofíticos.

Finalmente, los incendios con los códigos 23, 24, 25, 36 y 37 se localizan en diferentes sectores de las estribaciones meridionales del macizo del Moncayo y otras sie- 
Tabla 1: Relación de códigos ID por incendio, municipio, año y $\mathrm{n}^{\circ}$ de hectáreas afectadas y analizadas, seleccionados para los análisis de fPAR y LAI.

Table 1: Fire codes, municipality, year and number of hectares affected and analyzed, selected for the analysis of LAI and fPAR.

\begin{tabular}{|r|l|r|c|c|}
\hline \multicolumn{1}{|c|}{ ID } & \multicolumn{1}{|c|}{ Municipio } & Año & $\begin{array}{c}\text { Superficie } \\
\text { (ha) }\end{array}$ & $\begin{array}{c}\text { Superficie analizada } \\
\text { mediante MCD15A2 (ha) }\end{array}$ \\
\hline $\mathbf{6}$ & Las Peñas de Riglos & 1975 & 639 & 300 \\
\hline $\mathbf{7}$ & Nueno & 1977 & 809 & 200 \\
\hline $\mathbf{1 0}$ & Zuera & 1979 & 759 & 100 \\
\hline $\mathbf{1 1}$ & Nueno & 1980 & 523 & 100 \\
\hline $\mathbf{1 3}$ & Sabiñáñigo & 1981 & 762 & 300 \\
\hline $\mathbf{1 7}$ & Anzánigo & 1985 & 694 & 200 \\
\hline $\mathbf{1 9}$ & Las Peñas de Riglos & 1985 & 735 & 100 \\
\hline $\mathbf{2 3}$ & Fuentes de Jiloca & 1987 & 653 & 200 \\
\hline $\mathbf{2 4}$ & Ambel & 1991 & 1.847 & 600 \\
\hline $\mathbf{2 5}$ & Calcena & 1993 & 3.695 & 1.100 \\
\hline $\mathbf{2 6}$ & Sos del Rey Católico & 1994 & 8.078 & 2.900 \\
\hline $\mathbf{2 9}$ & Uncastillo & 1994 & 2.424 & 700 \\
\hline $\mathbf{3 1}$ & Las Peñas de Riglos & 2001 & 3.278 & 900 \\
\hline $\mathbf{3 2}$ & Zaragoza (CENAD) & 2004 & 696 & 100 \\
\hline $\mathbf{3 4}$ & Zuera y Castejón de Valdejasa & 2008 & 2.514 & 300 \\
\hline $\mathbf{3 6}$ & Jaulín & 2009 & 1.706 & 100 \\
\hline $\mathbf{3 7}$ & Terrer & 2009 & 679 & 3.200 \\
\hline $\mathbf{3 8}$ & Zaragoza (CENAD) & 2009 & 6.978 & \\
\hline
\end{tabular}

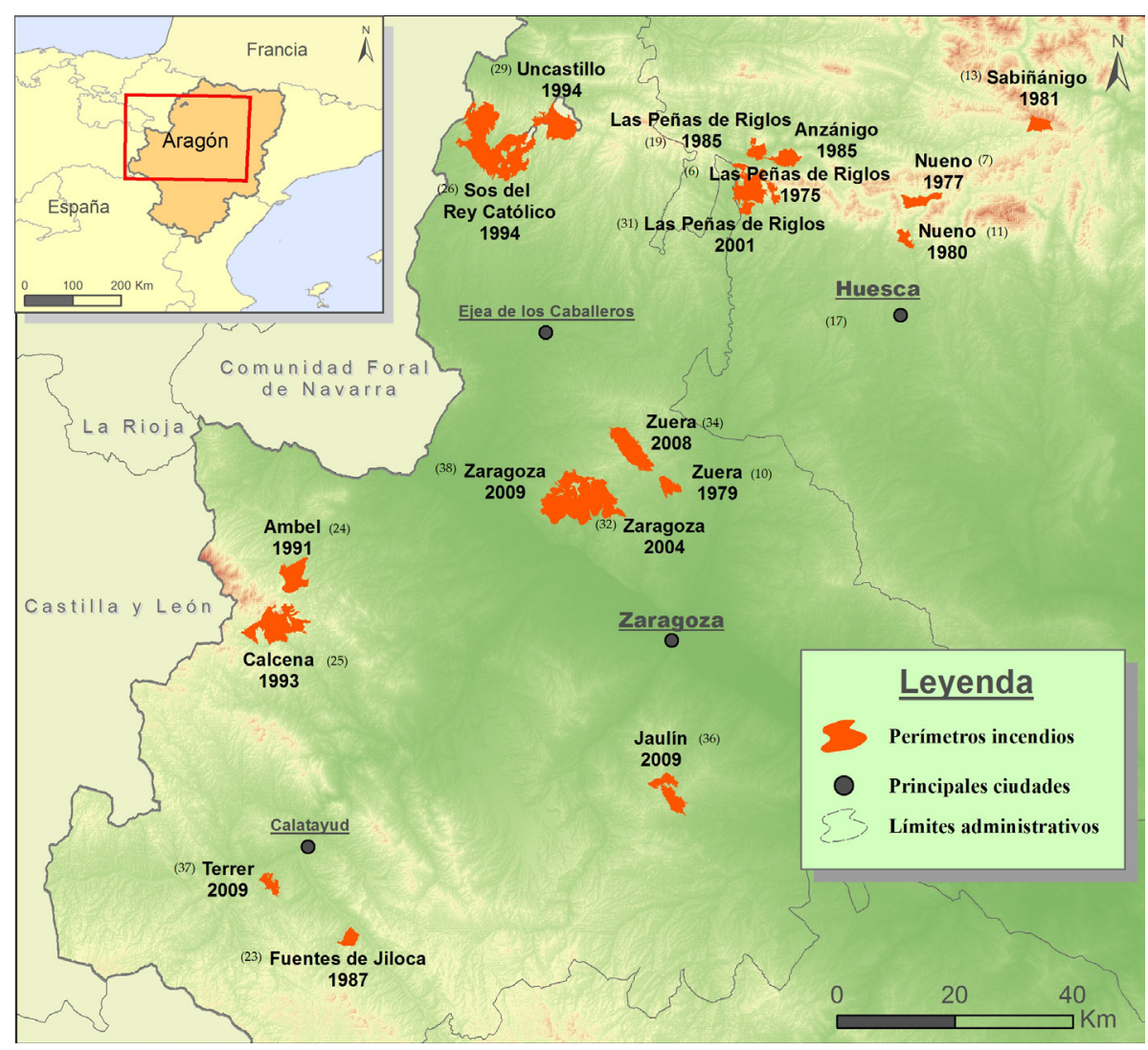

Figura 1: Mapa de localización de los incendios forestales con sus respectivas fechas y códigos. Fuentes cartográficas: IGN y SITAR Figure 1: Map of location of forest fires with their respective dates and codes. Cartographic sources: IGN and SITAR. 
rras zaragozanas. En este caso, las zonas afectadas estaban cubiertas de manera predominante por matorrales incluidos en la alianza mediterránea Rosmarino-Ericion, encinares y plantaciones de Pinus pinaster (Aiton) y $\mathrm{Pi}$ nus halepensis L. (Ruiz de la Torre, 1990b; Villar, 1990).

Desde el punto de vista de la estrategia reproductiva tras el fuego, esta amplia muestra de ecosistemas contiene especies vegetales con tres tipos básicos de respuesta al fuego (Pausas, 2012): (a) Reclutadores obligados dominados por $P$. halepensis, especie dotada de un extenso banco de semillas aéreo (serotinia) que posibilita una recolonización de tipo endógeno; (b) Sistemas pasivos de defensa, en este caso se trata de comunidades dominadas por $P$. nigra y $P$. sylvestris que mantienen una estrategia resistente al fuego mediante sistemas pasivos y germinación exógena; y (c) Rebrotadores obligados, reúnen a fanerófitos y macro-fanerófitos pertenecientes al género Quercus (Q. ilex, Q. gr. cerrioides, $Q$. coccifera) con estrategia de rebrote mediante yemas adventicias.

\subsection{Generación de la base de datos a partir del producto MCD13A2}

Una vez recabados todos los perímetros de los incendios de interés se procedió a la descarga de los datos MODIS a través del servidor Earth Explorer del USGS (http://earthexplorer.usgs.gov/). Tal y como se cita más arriba, al descargar los datos se seleccionó el tile o imagen de $17 \mathrm{~h}$ y $4 \mathrm{v}$ de la rejilla de la proyección sinusoidal de MODIS.

Se descargaron dos compuestos estacionales de 8 días, primera semana de mayo y septiembre, por considerarse claves en el registro del contraste entre la actividad fotosintética de la vegetación a finales de primavera y el descenso de la misma al final del verano, justo antes de que se generalicen las lluvias otoñales (López et al., 2007). Además, en septiembre la geometría de iluminación presenta menos problemas que durante los meses siguientes como consecuencia del descenso del ángulo de elevación solar.

Por otro lado, se utilizaron los parámetros de calidad (Quality flags -QC-) del propio producto con el propósito de eliminar posibles píxeles alterados por la presencia de nubes, sombras de nube, etc. A partir de aquí se tomarán las siguientes denominaciones para los compuestos y variables: $\mathrm{LAI}_{\text {sep }}$ y LAI ${ }_{\text {may }}$ para los compuestos de septiembre y mayo, respectivamente, del índice de área foliar; y fPAR y fPAR ${ }_{\text {may }}$ para los compuestos de la variable fracción de la radiación fotosintéticamente activa. Los valores que adquieren estos dos parámetros pueden oscilar entre 0 y 8 para el caso del LAI y entre 0 y 1 para fPAR.

\subsection{Perspectivas temporales de análisis $y$ procedimientos estadísticos}

La diferente información recopilada a partir de los productos MODIS se analiza en dos vertientes diferentes: (1) análisis estático de los valores de LAI/fPAR corres- pondientes a zonas quemadas a lo largo del periodo 1975 2010; (2) seguimiento multianual en 6 grandes incendios ocurridos en la década de 2000, representativos de condiciones ambientales diferentes.

(1) El análisis estático de los valores de LAI/fPAR (compuestos de 2010) referidos a zonas afectadas en diferentes años a lo largo del periodo (1976-2010), nos permite analizar el factor tiempo en la recuperación de los valores de LAI/fPAR. En los compuestos de 2010 quedan recogidos todos los incendios del periodo elegido.

Debido al hecho de que no se dispone de zonas quemadas en todos y cada uno de los años en este periodo, los diferentes incendios han sido agrupados en intervalos temporales en función del año que ocurrieron. A continuación se enumeran dichas agrupaciones: $\mathrm{I}_{1}=$ superficies quemadas con un año de antigüedad en relación con el último compuesto disponible (2010); $\mathrm{I}_{2}=$ superficies quemadas con dos años de antigüedad; $\mathrm{I}_{10}$ superficies quemadas entre $2 \mathrm{y}$ 10 años de antigüedad; $I_{20}=$ superficies quemadas entre 10 y 20 años de antigüedad; $I_{30}=$ superficies quemadas entre 20 y 30 años de antigüedad; $I_{35}=$ superficies quemadas con más de 35 años de antigüedad.

El perfil asimétrico de los intervalos se relaciona con el ajuste exponencial entre tiempo y NDVI descrito en muchos tipos de vegetación de tipo mediterránea (Viedma et al., 1997), caracterizado por un rápido incremento en los primeros años tras la perturbación seguido de etapas con un crecimiento más moderado, hasta llegar al comportamiento asintótico a medio plazo.

(2) El segundo de los análisis se trata de un estudio a mayor resolución temporal (análisis multianual) considerando de manera individual 6 grandes incendios producidos en la década de 2000 (a partir de 2002 se dispone de cartografía del producto MODIS MCD13A2). Este segundo análisis permite comprobar los efectos inmediatos del fuego sobre los valores de LAI/fPAR y caracterizar la respuesta de la vegetación, a una resolución temporal de mayor detalle, en zonas quemadas inscritas en diferentes marcos de referencia espacial de la región aragonesa.

Desde el punto de vista de los análisis estadísticos aplicados, mediante análisis de varianza (ANOVA) y la prueba post hoc de Tamhane T2, con gráficos de intervalos de confianza para la media, se comprueba si las medias de los valores de LAI/fPAR presentan diferencias estadísticamente significativas entre agrupaciones temporales. Además se realizan análisis de regresión simple, utilizando el coeficiente de determinación $\mathrm{r}^{2}$ como medida de ajuste, para identificar tendencias en el tiempo y el grado de ajuste entre los valores de fPAR y LAI.

\section{Resultados y discusión}

\subsection{Caracterización de superficies afectadas por incendio (1975-2010) mediante productos MCD15A2}

En relación con los niveles de fPAR/LAI recogidos en zonas quemadas de Aragón en el periodo comprendido entre 
1975 y 2010, en la Tabla 2 se recogen estadísticos descriptivos referentes a los compuestos de mayo y septiembre (fPAR $_{m a y / s e p}$ y LAI $_{m a y / s e p}$ ) de 2010. Dichos valores han sido extraídos en diferentes píxeles en función de la fecha en la que se produjo el fuego (desde hace un año, hasta píxeles que se vieron afectados por el fuego hace más de 35 años). En total la información analizada proviene de un total 127 píxeles que se corresponden con $127 \mathrm{~km}^{2}$ de área quemada procedentes de los 18 incendios considerados en este trabajo.

En lo relativo a los intervalos temporales que se han propuesto para el análisis conjunto de las superficies quemadas, los valores más bajos, tanto de LAI como de fPAR, los encontramos lógicamente en el intervalo $I_{1}$, un año después del incendio, en los compuestos de septiembre $\left(\mathrm{fPAR}_{\text {sep }}=0,16 ; \mathrm{LAI}_{\text {sep }}=0,24\right)$. Estos valores, por tanto, pueden ser representativos del grado de regeneración de las estructuras vegetales en el primer año tras el fuego; evidentemente, se trata de un valor medio proveniente de varias zonas quemadas sin tener en cuenta diferentes factores de carácter coyuntural y/o estructural, tales como la severidad o el tipo de formación vegetal afectada, respectivamente.
En el otro extremo, los valores más elevados (fPAR = 0,50 , en cualquiera de los compuestos; $\mathrm{LAI}=1,13 / 1,40$ para septiembre y mayo respectivamente) se corresponden con las superficies quemadas con mayor antigüedad $\left(\mathrm{I}_{35}\right)$. Aunque los valores en los intervalos extremos no son estrictamente comparables, dado que agrupan incendios diferentes en cuanto a las formaciones vegetales afectadas, contexto ambiental, etc., resulta significativo el hecho de que, 35 años tras el fuego, podamos encontrar valores que en el caso de fPAR triplican y en el caso de LAI quintuplican a los recogidos un año tras el fuego.

La mayor cuantía de los valores de LAI/fPAR en $\mathrm{I}_{30-35}$ puede interpretarse en términos de recuperación de la productividad, incremento del nivel de intercambio de los flujos de masas y energía en el sistema atmósfera-biosfera y, desde el punto de vista de la protección del suelo, mejora en los parámetros controladores de la interceptación de la lluvia. No obstante, estos valores deben ser interpretados con cierta cautela ya que numerosos trabajos de verificación realizados hasta la fecha sobre los productos MODIS alertan de la sobreestimación de los registros del producto MCD15A2 en comparación con observaciones de campo

Tabla 2: Estadísticos descriptivos de los valores de fPAR y LAI en la imagen de 2010, registrados en todas las zonas quemadas, por intervalos temporales.

Table 2: Descriptive statistics of the values of LAI and fPAR in the image of 2010, registered in all areas burned by intervals.

\begin{tabular}{|l|c|c|c|c|c|c|c|}
\cline { 2 - 8 } \multicolumn{1}{c|}{} & Intervalos & $\mathbf{N}$ & Media & Desv. típica & Mínimo & Máximo & Coef. var. (\%) \\
\hline$f P A R_{\text {sep }}$ & $\mathrm{I}_{1}$ & 36 & 0,16 & 0,01 & 0,13 & 0,20 & 8,38 \\
\hline$f P A R_{\text {sep }}$ & $\mathrm{I}_{2}$ & 13 & 0,30 & 0,07 & 0,16 & 0,43 & 24,65 \\
\hline$f P A R_{\text {sep }}$ & $\mathrm{I}_{10}$ & 10 & 0,37 & 0,06 & 0,24 & 0,46 & 15,41 \\
\hline$f P A R_{\text {sep }}$ & $\mathrm{I}_{20}$ & 53 & 0,36 & 0,11 & 0,18 & 0,70 & 31,38 \\
\hline$f P A R_{\text {sep }}$ & $\mathrm{I}_{30}$ & 8 & 0,43 & 0,08 & 0,33 & 0,60 & 19,77 \\
\hline$f P A R_{\text {sep }}$ & $\mathrm{I}_{35}$ & 7 & 0,48 & 0,08 & 0,35 & 0,55 & 16,94 \\
\hline$f P A R_{\text {mav }}$ & $\mathrm{I}_{1}$ & 36 & 0,20 & 0,07 & 0,07 & 0,40 & 35,00 \\
\hline$f P A R_{\text {mav }}$ & $\mathrm{I}_{2}$ & 13 & 0,45 & 0,08 & 0,35 & 0,65 & 17,71 \\
\hline$f P A R_{\text {mav }}$ & $\mathrm{I}_{10}$ & 10 & 0,42 & 0,04 & 0,37 & 0,47 & 9,72 \\
\hline$f P A R_{\text {may }}$ & $\mathrm{I}_{20}$ & 53 & 0,45 & 0,11 & 0,22 & 0,69 & 24,05 \\
\hline$f P A R_{\text {mav }}$ & $\mathrm{I}_{30}$ & 8 & 0,39 & 0,09 & 0,27 & 0,57 & 23,16 \\
\hline$f P A R_{\text {mav }}$ & $\mathrm{I}_{35}$ & 7 & 0,50 & 0,06 & 0,40 & 0,56 & 11,65 \\
\hline$L A I_{\text {sep }}$ & $\mathrm{I}_{1}$ & 36 & 0,24 & 0,05 & 0,20 & 0,40 & 22,98 \\
\hline$L A I_{\text {sep }}$ & $\mathrm{I}_{2}$ & 13 & 0,58 & 0,14 & 0,30 & 0,80 & 24,67 \\
\hline$L A I_{\text {sep }}$ & $\mathrm{I}_{10}$ & 10 & 0,69 & 0,18 & 0,40 & 1,10 & 25,97 \\
\hline$L A I_{\text {sep }}$ & $\mathrm{I}_{20}$ & 53 & 0,72 & 0,36 & 0,30 & 2,00 & 50,38 \\
\hline$L A I_{\text {sep }}$ & $\mathrm{I}_{30}$ & 8 & 0,90 & 0,26 & 0,60 & 1,40 & 28,48 \\
\hline$L A I_{\text {sep }}$ & $\mathrm{I}_{35}$ & 7 & 1,13 & 0,29 & 0,60 & 1,40 & 25,94 \\
\hline$L A I_{\text {mav }}$ & $\mathrm{I}_{1}$ & 36 & 0,39 & 0,18 & 0,10 & 1,10 & 47,62 \\
\hline$L A I_{\text {mav }}$ & $\mathrm{I}_{2}$ & 13 & 1,22 & 0,32 & 0,70 & 1,90 & 25,99 \\
\hline$L A I_{\text {mav }}$ & $\mathrm{I}_{10}$ & 10 & 0,97 & 0,20 & 0,80 & 1,40 & 20,65 \\
\hline$L A I_{\text {may }}$ & $\mathrm{I}_{20}$ & 53 & 1,15 & 0,41 & 0,40 & 2,20 & 35,39 \\
\hline$L A I_{\text {mav }}$ & $\mathrm{I}_{30}$ & 8 & 0,98 & 0,32 & 0,50 & 1,40 & 32,32 \\
\hline$L A I_{\text {mav }}$ & $\mathrm{I}_{35}$ & 7 & 1,40 & 0,31 & 1,00 & 1,90 & 21,82 \\
\hline & & & & & & & \\
\hline
\end{tabular}


(Steinberg et al., 2006). Durá et al. (2012) identificaron una sobreestimación del $38 \%$ durante el año fenológico 2009-2010 en ambientes de dehesa en el que se producen dinámicas de LAI muy acusadas debido a la contribución de la componente herbácea de estos ecosistemas.

Esta disfunción se relaciona especialmente con los problemas de clasificación de los tipos de cubierta (Serbin et al., 2013) ya que, una mala asignación del bioma dentro de los algoritmos de LAI/fPAR, puede introducir errores superiores al 20\% (Myneni et al., 2002). En este sentido, a nivel estacional, se han identificado diferencias estadísticamente significativas ( $p$-value $<0,05$ ) en los valores de fPAR y LAI, entre los compuestos de mayo y septiembre, presentándose siempre guarismos más elevados en los compuestos de mayo (1,5 veces mayores en el caso del LAI y 1,2 en el caso de fPAR), al situarse en la fase primaveral del ciclo fenológico de la vegetación, momento en el que el desarrollo y la complejidad de su estructura foliar es mayor.

Por otro lado, hay que reseñar la alta variabilidad que presentan los valores registrados por intervalo temporal debido (1) a la heterogeneidad de las formaciones vegetales afectadas y de sus estrategias reproductivas; (2) a las diferencias en la coyuntura pluvio-térmica experimentada por cada zona quemada; y (3) al hecho de que en muchas zonas se han aplicado medidas de restauración hidrológico forestal. Es el caso de lo acontecido en algunos sectores de los incendios de Las Peñas de Riglos (cod.: 31), Zuera y Castejón de Valdejasa (cod.: 34), tal y como se ha podido constatar a raíz de la consulta en la Dirección General de Gestión Forestal (Departamento de Agricultura, Ganadería y Medio Ambiente del Gobierno de Aragón). La heterogeneidad de las varianzas en cada intervalo, en especial en el $\mathrm{I}_{20}$ que agrupa el mayor número de incendios, determinó que utilizáramos la prueba de Tamhane T2 para identificar la existencia de diferencias significativas entre intervalos.

Sin embargo, el gráfico de intervalos de confianza para la media que resume la distribución de los valores de fPAR dentro de las agrupaciones definidas, recogido en la Figura 2, pone de manifiesto la existencia de un patrón temporal en la distribución de los valores de fPAR a lo largo de los años de regeneración según la estación de los compuestos. En el caso de septiembre, se reconoce visualmente que no existen diferencias significativas entre los intervalos más alejados en el tiempo, aunque los valores son claramente superiores conforme aumenta la antigüedad, produciéndose un cambio de nivel entre los intervalos $\mathrm{I}_{10}-\mathrm{I}_{20}$ (entre 2 y 20 años de antigüedad) con los intervalos $\mathrm{I}_{30}-\mathrm{I}_{35}(<30$ años de antigüedad $)$.

En este sentido, en incendios acontecidos en la década de los ochenta en el área prepirenaica occidental de Aragón, algunos de los cuales han sido considerados en este estudio, se ha descrito que, aunque 10-15 años no constituyen una franja temporal suficiente para que las formaciones vegetales afectadas se recuperen de manera integral, es suficiente tiempo para proporcionar buenos niveles de recubrimiento vegetal, así como cierta correspondencia fisionómico-florística entre las comunidades

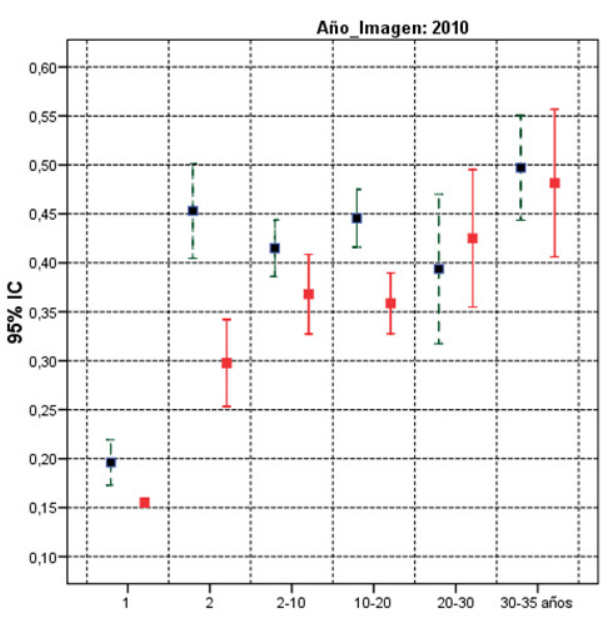

I PPAR_mayo
I PPAR_sep

Figura 2: Distribución de los valores de $\mathrm{fPAR}_{\text {may/sep }}$ (intervalos de confianza para la media) de la imagen de 2010 por intervalos temporales.

Figure 2: Distribution of the values of fPARmay/sep (confidence intervals for the mean) of the image of 2010 by intervals.

regeneradas y sus antecedentes (Pérez-Cabello, 2002). Indistintamente de la estrategia regenerativa de las comunidades afectadas, en estas primeras fases de regeneración son dominantes los estratos de vegetación herbácea y las leñosas inferiores a $0,5 \mathrm{~m}$ pudiendo cubrir por término medio el 50,3\% de los nuevos espacios regenerados tras el fuego.

En el caso de mayo, los valores, como se ha dicho más arriba, presentan guarismos más elevados aunque se reproduce el mismo patrón temporal descrito para septiembre, excepto en el caso del $\mathrm{I}_{2}$ que recoge valores inusuales y extraordinariamente altos. Ese último extremo se relaciona con la contribución de otras cubiertas (cultivos de cereal de secano) en los valores de LAI/fPAR, como consecuencia de la resolución espacial del producto MODIS y la estructura del paisaje de zonas quemadas situadas en la Depresión del Ebro (redes dendriformes de valles ocupados por cereal, rodeados de masas forestales). Por tanto, todo indica que, en zonas de gran heterogeneidad de cubiertas, los compuestos de otoño recogerían de manera más real el proceso de recuperación vegetal tras el fuego.

En la Figura 3 se representan los diagramas de dispersión correspondientes a las relaciones entre los valores de fPAR/LAI (compuestos de septiembre y mayo) y el tiempo trascurrido tras el fuego expresado en número de años. Los puntos representan a los valores medios y los errores típicos de las superficies quemadas cada año. Si bien los $\mathrm{r}^{2}$ obtenidos son bajos debido a las peculiaridades de cada uno de los incendios considerados (entre 0,49 y 0,29 ), se identifica claramente una tendencia positiva en la que los valores de fPAR y, en menor medida LAI, aumentan en función del número de años tras el fuego. De tal modo que los incendios con mayor tiempo para su recuperación presentan en general valores más elevados. La correlación es más acusada en los compuestos de septiembre de- 

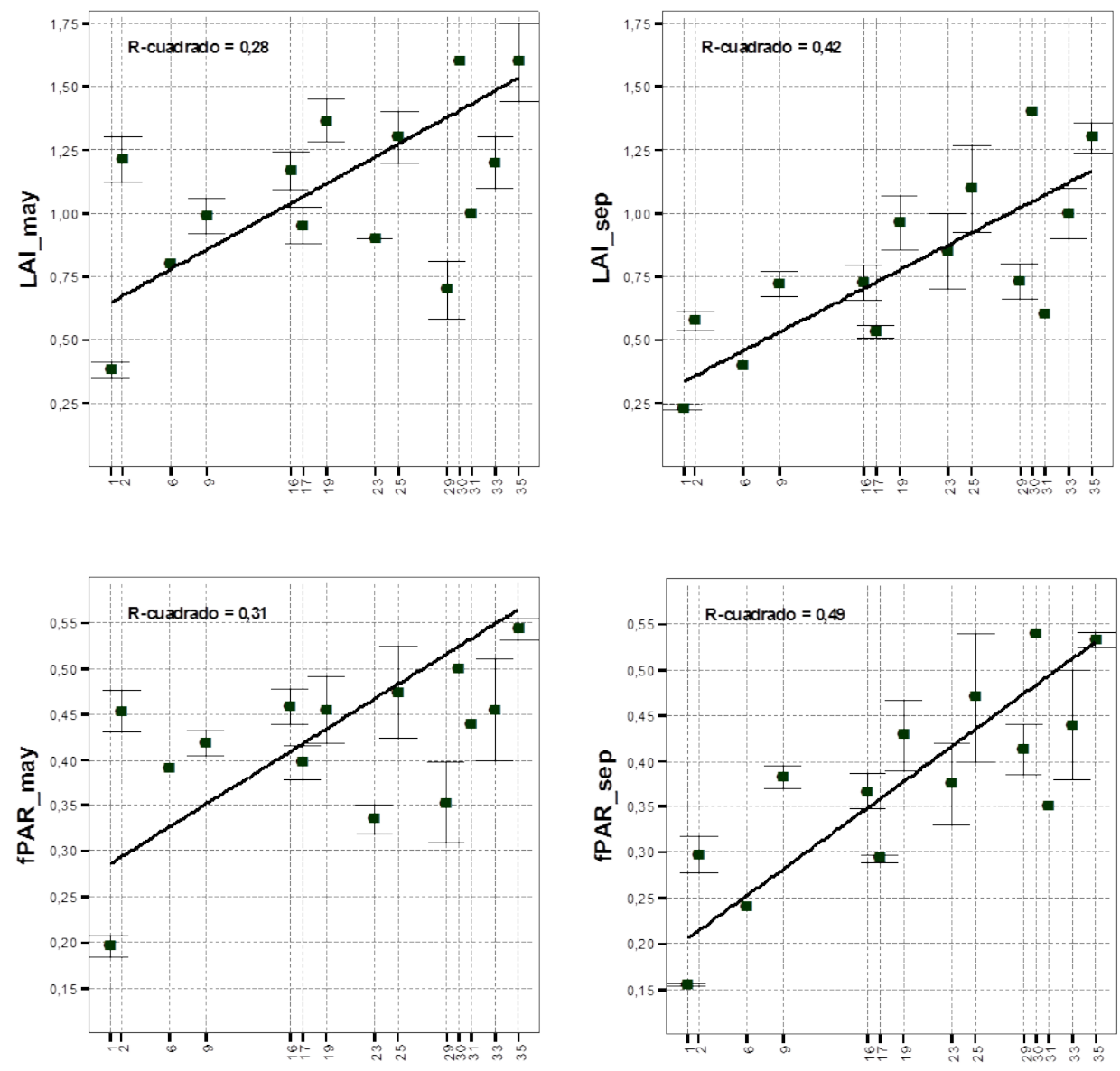

Figura 3. Distribución del error típico de la media de los valores de fPAR/LAI para los compuestos de septiembre y mayo en relación con el número de años tras el fuego analizado en la imagen de 2010.

Figure 3. Distribution of the standard error of the mean of the values of fPAR/LAI for compounds of September and May in relation to the number of years after the fire, scanned the image of 2010.

bido, especialmente, a su mejor ajuste en relación a los meses en los que suelen registrarse los incendios forestales, siendo muy similar la tendencia, independientemente de que se utilice fPAR o LAI.

Cabe preguntarse cómo se relacionan estos dos parámetros en las zonas quemadas. Físicamente la relación entre fPAR y LAI es exponencial inversa y muy dependiente del ángulo cenital solar (Jones \& Vaughan 2010). En las zonas analizadas afectadas por incendio, los resultados, considerando cualquiera de los compuestos estacionales -mayo y septiembre- de las fechas disponibles y cuatro marcos geográficos, se identifica una relación de tipo lineal con $r^{2}$ superiores a 0,85 (Figura 4), debido probablemente al menor rango de los valores. Por tanto, aunque fPAR y LAI conceptualmente se refieren a variables biofísicas distintas del dosel vegetal, se encuentran estrechamente relacionadas (Zarate-Valdez et al., 2012; Glenn et al., 2008).

Si bien ambas variables son muy dependientes de las condiciones ambientales, LAI es una variable de mayor complejidad que se vincula de modo más directo a la capacidad fotosintética de las formaciones vegetales. Prueba de la mayor complejidad del LAI reside en el hecho de que mediante la inversión de la ecuación para la predicción de la radiación fotosintéticamente activa transmitida bajo el dosel (Norman \& Campbell, 1989) es posible transformar fPAR en LAI siempre y cuando se disponga de datos de absortividad foliar y de los coeficientes de extinción de la radiación. Estos últimos dependen a su vez de las funciones de distribución angular de las hojas.

\subsection{Dinámica multianual de los valores de LAI en zonas quemadas (2001-2010)}

El análisis de la dinámica tras el fuego a mayor resolución temporal (año por año) se ha realizado para el periodo 2001-2010 en 6 incendios de la base de datos ocurridos en dicho intervalo temporal (Las Peñas de Riglos, CENAD (2), Zuera y Castejón de Valdejasa, Jaulín y Terrer), representativos además de los marcos geográficos contemplados en la zona de estudio. Para estos incendios se dispone de información sobre la situación previa y posterior a la perturbación, excepto en uno de los casos (Incendio de las Peñas de Riglos). 

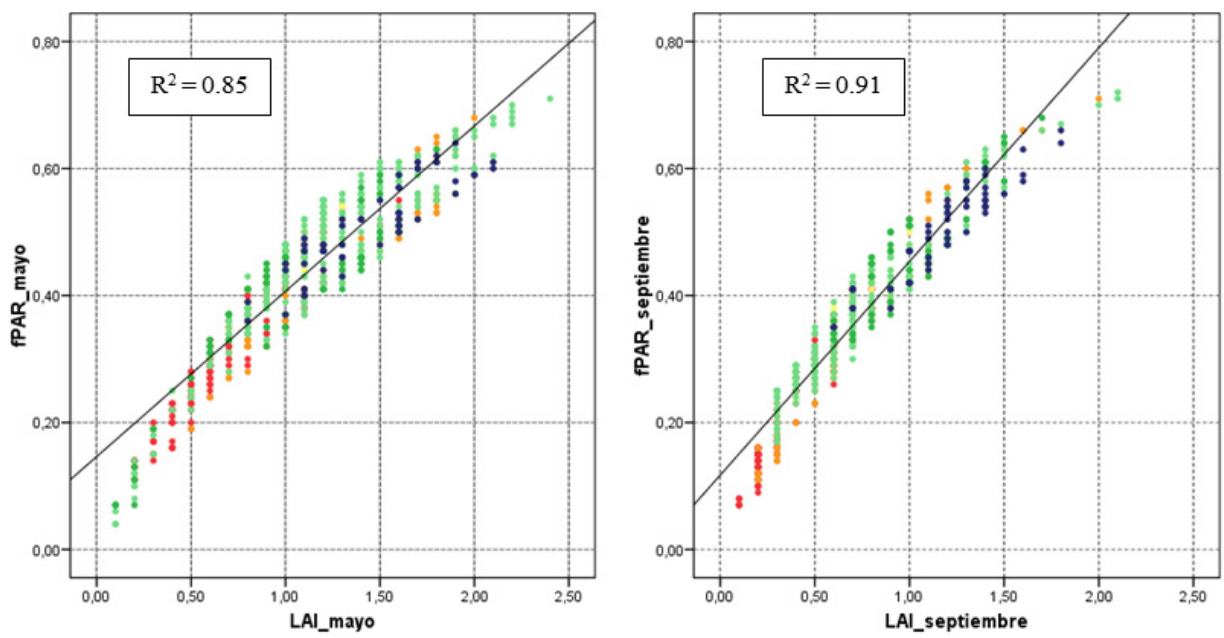

Figura 4: Gráficos de dispersión y líneas de ajuste entre fPAR y LAI por compuesto estacional. En color se muestra los valores por intervalos temporales.

Figure 4: Scatter charts and line adjustment between LAI and fPAR seasonal composite. Color shows the values by intervals.

La Figura 5 y la Tabla 3 recogen la evolución temporal de los valores de LAI en el compuesto de septiembre. El análisis se ha restringido exclusivamente con este compuesto debido a su mayor proximidad a las fechas concretas del fuego, todos son incendios de verano, y al hecho de que el compuesto de mayo se ajusta peor al análisis de la evaluación de las repercusiones inmediatas del fuego en los valores de LAI. Del mismo modo, una vez analizada la correlación entre los valores de LAI y fPAR (apartado 3.1), el análisis se ha restringido sólo a los valores de LAI.

El primero de los gráficos contenidos en la Figura 5 (arriba a la izquierda) representa la trayectoria multianual del incendio de las Peñas de Riglos de 2001 (código 31), perteneciente al marco geográfico de las Sierras occidentales del Prepirineo oscense, dominado en este caso por comunidades de carácter submediterráno (Quercus gr. cerrioides y Buxus sempervirens), plantaciones de Pinus nigra y matorrales.

En este caso, la curva sólo recoge el proceso de recuperación de los valores de LAI transcurridos dos años tras el fuego debido a que el incendio tuvo lugar en 2001 y la serie de datos que se maneja comienza en 2003, ya que el compuesto utilizado combina la información espectral procedente de dos satélites, Terra y Aqua, siendo este último lanzado más tarde, en 2002. En general la curva muestra gran estabilidad interanual, a pesar de la posible influencia en los valores de los trabajos de apeo y extracción de la madera quemada y de las tareas de restauración de la vegetación, a las que se vio sometido desde el año siguiente al fuego; al menos en los 2/3 de la superficie afectada (2.212 ha). No obstante, destaca el descenso de los valores en 2005, como consecuencia de los efectos de la sequía del año hidrológico 2004-2005. A partir de un simple análisis de la serie mensual de datos de precipitación de la estación de Huesca (Red de Estaciones Agro-meteorológicas del SIAR -MAGRA-
MA-), próxima al incendio de las Peñas de Riglos, se recoge en ese año una reducción del $33 \%$ respecto a la media histórica.

El gráfico con el código 32 representa la evolución del incendio de 2004 que tuvo lugar dentro del CENAD, dominado por formaciones de carácter subarbustivo mediterráneo (Rosmarinus officinalis, Genista scorpius, etc.) con pastizales xerofíticos. En la curva se advierte el descenso provocado por el fuego (decremento del $57 \%$ del valor previo de LAI) y la asténica recuperación durante los años siguientes cuyo inicio hay que situarlo en 2006, dos años después del fuego. Dicho retraso podría responder a las situaciones de estrés hídrico de 2005 en esta zona. Este mismo proceso de ralentización de la regeneración con motivo de la menor cantidad de precipitación en 2005 fue descrito en la estación experimental de Peñaflor (EEP) (cercana CENAD), en donde se realizó un fuego controlado sobre formaciones vegetales similares a las de la zona del CENAD (Pérez-Cabello et al., 2012), recogiéndose un $25 \%$ menos de precipitación media anual.

El gráfico con el código 34 se corresponde con el incendio de Zuera y Castejón de Valdejasa de 2008, que afectó en su mayoría a una masa de pinar de Pinus halepensis con Q. coccifera. El valor de 2008 recoge el impacto del fuego, mientras que los registros de $2009 \mathrm{y}$ 2010 representan la recuperación de los valores de LAI. El incremento de los valores de LAI en este caso puede explicarse por la eficacia de la estrategias activas de respuesta al fuego que detentan las dos especies principales afectadas: dispersión de propágulos en el caso de Q. coccifera, y germinación en el caso de $P$. halepensis, ya documentadas ampliamente en zonas incendiadas próximas al incendio de 2008 (Vicente-Serrano et al., 2011; Tanase et al., 2011).

Por otro lado, en este incendio hay que destacar la alta variabilidad que recogen los valores de LAI en los años previos al fuego, así como el inexplicable descenso de 
31

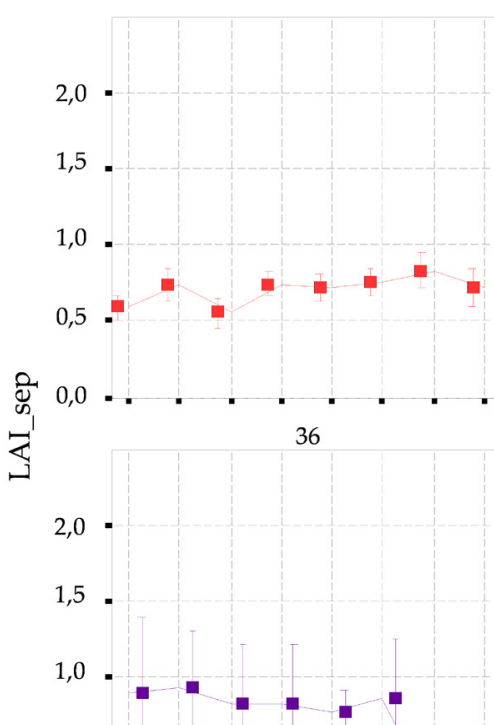

32

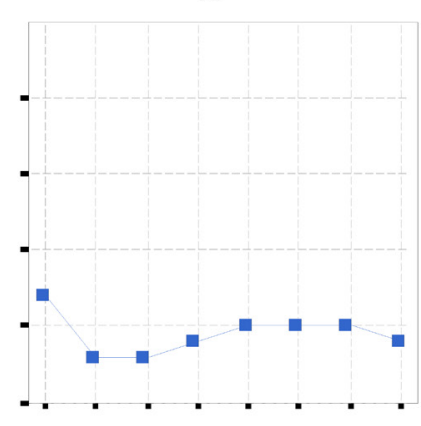

37

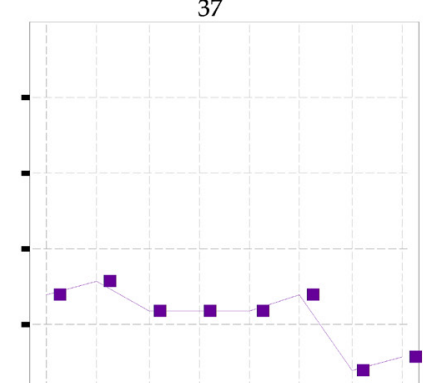

34

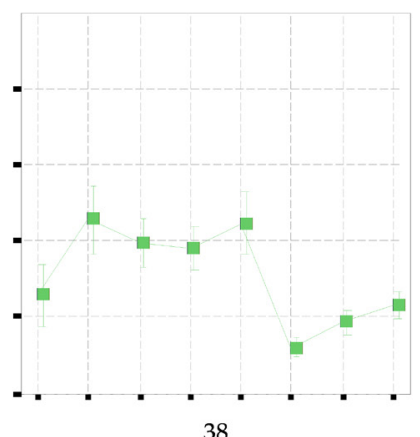

38

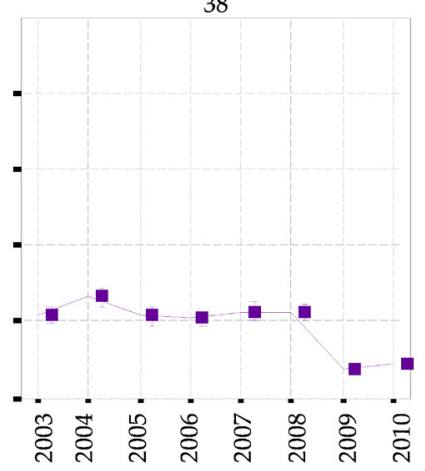

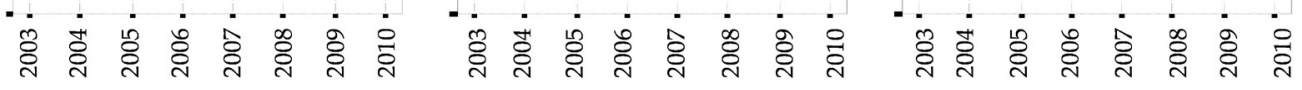

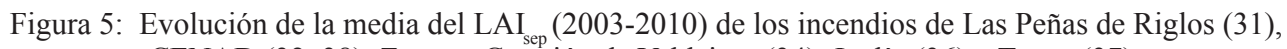
CENAD $(32,38)$, Zuera y Castejón de Valdejasa (34), Jaulín (36) y Terrer (37).

Figure 5: Evolution of the average of the LAIsep (2003-2010) of the fires: Las Peñas de Riglos (31), CENAD (32, 38), Zuera y Castejón de Valdejasa (34), Jaulin (36) y Terrer (37).

Tabla 3: Estadísticos descriptivos de la evolución temporal de los valores de LAI.

Table 3: Descriptive statistics of the temporal evolution of the values of LAI.

\begin{tabular}{|c|c|c|c|c|c|c|c|c|c|c|c|c|}
\hline \multirow{2}{*}{ LAIsep } & \multicolumn{4}{|c|}{ Las Peñas de Riglos (31) } & \multicolumn{4}{|c|}{ CENAD (32) } & \multicolumn{4}{|c|}{ Zuera y Castejón V. (34) } \\
\hline & Media & Des. Típ. & Min. & Máx. & Media & Des. Típ. & Min. & Máx. & Media & Des. Típ. & Min. & Máx. \\
\hline 2003 & 0,59 & 0,11 & 0,50 & 0,80 & 0,70 & . & 0,70 & 0,70 & 0,65 & 0,34 & 0,20 & 1,10 \\
\hline 2004 & 0,73 & 0,14 & 0,60 & 1,00 & 0,30 & . & 0,30 & 0,30 & 1,14 & 0,38 & 0,50 & 2,00 \\
\hline 2005 & 0,56 & 0,12 & 0,40 & 0,80 & 0,30 & . & 0,30 & 0,30 & 0,99 & 0,27 & 0,50 & 1,50 \\
\hline 2006 & 0,74 & 0,10 & 0,60 & 0,90 & 0,40 & . & 0,40 & 0,40 & 0,95 & 0,23 & 0,50 & 1,30 \\
\hline 2007 & 0,72 & 0,12 & 0,60 & 0,90 & 0,50 & . & 0,50 & 0,50 & 1,12 & 0,33 & 0,40 & 1,70 \\
\hline 2008 & 0,76 & 0,12 & 0,60 & 1,00 & 0,50 & . & 0,50 & 0,50 & 0,30 & 0,10 & 0,20 & 0,50 \\
\hline 2009 & 0,83 & 0,16 & 0,70 & 1,10 & 0,50 & . & 0,50 & 0,50 & 0,47 & 0,13 & 0,30 & 0,60 \\
\hline \multirow[t]{3}{*}{2010} & 0,72 & 0,16 & 0,60 & 1,10 & 0,40 & . & 0,40 & 0,40 & 0,58 & 0,14 & 0,30 & 0,80 \\
\hline & \multicolumn{4}{|c|}{ Jaulín (36) } & \multicolumn{4}{|c|}{ Terrer (37) } & \multicolumn{4}{|c|}{ CENAD (38) } \\
\hline & Media & Des. Típ. & Min. & Máx. & Media & Des. Típ. & Min. & Máx. & Media & Des. Típ. & Min. & Máx. \\
\hline 2003 & 0,90 & 0,20 & 0,70 & 1,10 & 0,70 & . & 0,70 & 0,70 & 0,55 & 0,15 & 0,30 & 0,80 \\
\hline 2004 & 0,93 & 0,15 & 0,80 & 1,10 & 0,80 & . & 0,80 & 0,80 & 0,66 & 0,17 & 0,30 & 1,10 \\
\hline 2005 & 0,83 & 0,15 & 0,70 & 1,00 & 0,60 & . & 0,60 & 0,60 & 0,54 & 0,16 & 0,20 & 0,90 \\
\hline 2006 & 0,83 & 0,15 & 0,70 & 1,00 & 0,60 & . & 0,60 & 0,60 & 0,52 & 0,14 & 0,20 & 0,80 \\
\hline 2007 & 0,77 & 0,06 & 0,70 & 0,80 & 0,60 & . & 0,60 & 0,60 & 0,57 & 0,16 & 0,20 & 0,90 \\
\hline 2008 & 0,87 & 0,15 & 0,70 & 1,00 & 0,70 & . & 0,70 & 0,70 & 0,56 & 0,15 & 0,20 & 0,90 \\
\hline 2009 & 0,20 & 0,00 & 0,20 & 0,20 & 0,20 & . & 0,20 & 0,20 & 0,19 & 0,05 & 0,10 & 0,30 \\
\hline 2010 & 0,27 & 0,06 & 0,20 & 0,30 & 0,30 & . & 0,30 & 0,30 & 0,23 & 0,05 & 0,20 & 0,40 \\
\hline
\end{tabular}


2003. En este sentido, la singular estructura del paisaje de este sector, caracterizada por sistemas dendriformes de estrechos valles ocupados por cultivos de cereal de invierno e interfluvios cubiertos por arboledas de $P$. halepensis, podría explicar dicha variabilidad, en relación también con la resolución espacial de los productos MODIS. A pesar de que en septiembre la campaña cerealista ha finalizado, el diferente estado de los cultivos en relación a los restos de la siega y la colonización de vegetación natural, podría influir de manera significativa.

La fila inferior de la figura 5 recoge la evolución en tres incendios que tuvieron lugar en 2009. El impacto del fuego queda registrado en el acusado descenso en los valores de $\mathrm{LAI}_{\text {sep }}$ en el compuesto de 2009. Antes del fuego, destaca la estabilidad de los valores de $\mathrm{LAI}_{\text {sep }}$, sólo interrumpida por un leve repunte en el año 2004. En los tres casos, a pesar de partir de niveles previos al fuego diferentes, como consecuencia de que representan marcos geográficos distintos (matorrales suarbustivos mediterráneos y pastizales xerofíticos -38-, pinares de halepensis -36- y matorrales acidófilos -37), los valores tras el fuego presentan magnitudes similares, en torno a 0,20 , siendo significativamente diferentes a los de los años previos al fuego, mostrando un descenso del orden del $75 \%$.

Por tanto, las líneas de evolución presentadas a esta escala temporal reproducen los niveles de impacto y recuperación del índice de área foliar de la vegetación tras el fuego en diferentes formaciones vegetales. El descenso de los valores tras el fuego puede aportar nuevas claves de interpretación biofísica en relación con las mediciones de la severidad, aspecto muy importante desde el punto de vista de la recuperación de las zonas quemadas. Asimismo, las magnitudes en el incremento permiten cuantificar y determinar espacialmente el grado de recomposición de las funciones eco-fisiológicas de la vegetación en su relación con la atmósfera.

Por otro lado, un análisis más exhaustivo de diferentes factores que hayan contribuido de modo significativo en los valores de LAI/fPAR a lo largo del tiempo (termopluviometría, severidad del fuego, estrategia reproductiva), así como de información de campo, proporcionaría nuevos elementos de análisis para interpretar, de modo más preciso, los valores y los patrones espacio/temporales de la información derivada del producto MCD13A2 de MODIS.

\section{Conclusiones}

Los estudios en los que se utilizan imágenes de satélite para obtener cartografía de variables estructurales y eco-fisiológicas a escala regional son muy numerosos en la literatura. En la mayoría de los casos se sirven de índices de vegetación como consecuencia de la relación entre estos y variables como el LAI o la concentración de clorofila (Cohen et al., 2003).

El producto MCD15A2 de MODIS proporciona directamente valores de LAI o fPAR a partir de datos de teledetección. Sin embargo, su utilización en el campo de los análisis de los procesos de regeneración postincendio no es tan dilatada como en el caso de otros satélites, caso del programa Landsat (Iwata, et al., 2013; Frazier et al., 2013; Romo et al., 2012). Su menor resolución espacial, la disponibilidad de un archivo más limitado en el tiempo y los problemas de sobreestimación documentados en la literatura justifican en cierta medida su escasa utilización en el contexto de los estudios sobre zonas quemadas.

En este trabajo se utiliza el producto MDC15A2 (LAI/ fPAR) de MODIS para la evaluación de las zonas afectadas por incendios forestales en Aragón. Estas variables, altamente correlacionadas entre sí, proporcionan información clave en estudios de eco-fisiología de los sistemas forestales, pudiendo ser utilizadas también en el análisis de la recuperación de zonas quemadas. Sin embargo, su interpretación no está exenta de problemas debido al dinamismo de los valores en relación a las condiciones de la época de medición y a la resolución espacial del producto, ya que puede recoger una gran diversidad de situaciones.

Se ha identificado una relación directa entre el tiempo transcurrido desde el fuego y los valores de LAI/fPAR. De tal modo que los valores promedio más elevados de LAI $(>1)$, es decir, las estructuras de vegetación más complejas las encontramos en incendios con más de 35 años de antigüedad. Por otra parte, justo después del fuego se ha podido comprobar que los valores de LAI/fPAR experimentan un acusado descenso $(\sim 75 \%)$ como consecuencia de la combustión de la vegetación y la consiguiente reducción de la interceptación de radiación solar.

\section{Agradecimientos}

Este trabajo ha sido realizado gracias al proyecto de investigación "Incendios forestales y modelos predictivos de vulnerabilidad ecológica frente al fuego: medidas de restauración y aplicaciones en escenarios de cambio climático" (Código: 247182) (Convenio de Colaboración entre el Gobierno de Aragón y la Obra Social "La Caixa" para el desarrollo del Programa de Investigación y Conservación de los Espacios Naturales gestionados por el Gobierno de Aragón).

Para el desarrollo del mismo se ha contado además con una beca de colaboración (6613955, del Ministerio de Educación, Cultura y Deporte) desarrollada conjuntamente entre el Departamento de Geografía y Ordenación del Territorio (Universidad de Zaragoza) y el Grupo Geoforest-IUCA durante el periodo 2012/2013.

\section{Referencias}

Chen, J. M. \& Black, T. A., 1992. Defining leaf area index for non-flat leaves. Plant, Cell \& Environment, 15 (4): 421-429. http://dx.doi.org/10.1111/j.1365-3040.1992.tb00992.x.

Chen, J. M., Rich, P. M., Gower, S. T., Norman, J. M. \& Plummer, S. 1997. Leaf area index of boreal forests: Theory, techniques and measurements. Journal of Geophysical Research. Atmospheres, 102: 429-443. http://dx.doi.org/10.1029/97JD01107. 
Chuvieco, E., Giglio, L. \& Justice, C. O., 2008. Global Characterization of Fire Activity: Towards Defining Fire Regimes From Earth Observation Data. Global Change Biology, 14: 14881502. http://dx.doi.org/10.1111/j.1365-2486.2008.01585.x.

Cohen, W. B., Maierpserger, T. K., Gower, S. T., \& Turner, D. P. 2003. An improved strategy for regression of biophysical variables and Landsat ETM+ data. Remote Sensing of Environment, 84: 561-571. http://dx.doi.org/10.1016/S0034-4257(02)00173-6.

Díaz-Delgado, R. \& Pons, X., 2001. Spatial patterns of forest fires in Catalonia (NE of Spain) along the period 1975-1995: analysis of vegetation recovery after the fire. Forest Ecology and Management, 147: 67-74. http://dx.doi.org/10.1016/S0378-1127(00)00434-5.

Durá, E., Mendiguren, G., Pacheco, J., Martín, M. P., Ria-o, D., Iturrate, M., Gimeno, C. \& Carrara, A., 2013. Validación de productos MODIS relacionados con la estimación de flujos de carbono en un ecosistema de dehesa. GeoFocus, 13(1): 291-310.

Fensholt, R., Sandholt, I. \& Rasmussen, M. S., 2004. Evaluation of MODIS LAI, fAPAR and the relation between FAPAR and NDVI in a semi-arid environment using in situ measurements. Remote Sensing of Environment, 91: 490-507. http://dx.doi.org/10.1016/j.rse.2004.04.009.

Frazier, A. E., Renschler, C. S. \& Miles, S. B., 2013. Evaluating post-disaster ecosystem resilience using MODIS GPP data. International Journal Applied Earth Observation, 21: 43-52. http://dx.doi.org/10.1016/j.jag.2012.07.019.

Gao, B.-C., 1996. NDWI A Normalized Difference Water Index for Remote Sensing of Vegetation. Liquid Water From Space. Remote Sensing of Environment, 58: 257-266. $\mathrm{http} / / / \mathrm{dx}$. doi.org/10.1016/S0034-4257(96)00067-3.

Gilabert, M. A., González-Piqueras, J. \& García-Haro, J., 1997. Acerca de los índices de vegetación. Revista de Teledetección, 8: 35-45.

Glenn, E., Huete, A. R., Nagler, P. L. \& Nelson, S. G., 2008. Relationship between remotely-sensed vegetation indices, canopy attributes and plant physiological processes: what vegetation indices can and cannot tell us about the landscape. Sensors, 8: 2136-2160. http://dx.doi.org/10.3390/ s8042136.

Huete, A. R., 1988. A Soil-Adjusted Vegetation Index (SAVI). Remote Sensing of the Environment, 25: 295-309. $\mathrm{http}: / / \mathrm{dx}$.doi.org/10.1016/0034-4257(88)90106-X.

Iwata, H., Ueyama, M., Iwama, C. \& Harazono Y., 2013. A variation in the fraction of absorbed photosynthetically active radiation and a comparison with MODIS data in burned black spruce forests of interior Alaska. Polar Science, 7: 113-124. http://dx.doi.org/10.1016/j.polar.2013.03.004.

Jones, H. G. \& Vaughan, R. A., 2010. Remote sensing of vegetation. Principles, techniques and applications. New York, USA: Oxford University Press.

Justice, C. O., Townshend, J. R. G., Vermote, E. F., Masuoka, E., Wolfe, R. E. \& El-Saleous, N., 2002. An overview of MODIS land data processing and product status. Remote Sensing of Environment, 83: 3-15. http://dx.doi.org/10.1016/ S0034-4257(02)00084-6.

Knyazikhin, Y., Martonchik, J. V., Myneni, R. B., Diner, D. J. \& Running, S. W., 1998. Synergistic algorithm for estimating vegetation canopy leaf area index and fraction of absorbed photosynthetically active radiation from MODIS and MISR data. Journal Geophysics Research, 103: 32257-32275. http://dx.doi.org/10.1029/98JD02462.

López, F., Cabrera, M. \& Cuadrat, J. M., 2007. Atlas climático de Aragón. Gobierno de Aragón. ISBN 978-84-8380-071-3.

Mooney, H. A. \& Hobbs, R. H., 1986. Resilience at the individual plant level. In: Dell, D., Hopkins, A.J.; Lamont, B.B. (eds) Resilience in Mediterrenean type ecosystems, 65-82 pp. La Haya. http://dx.doi.org/10.1007/978-94-009-4822-8_5.
Myneni, R., Knyazikhin, Y., Glassy, J., Votava, P. \& Shabanov, N., 2003. FPAR, LAI (ESDT: MOD15A2) 8-day Composite NASA MODIS Land Algorithm, User's Guide. MODIS website.

Myneni, R. B., Hoffman, S., Knyazikhin, Y., Privette, J. L., Glassy, J., Tian, Y. Wang, Song, X., Zhang, Y., Smith, G. R., Lotsch, A., Friedl, M., Morisette, J. T., Votava, P., Nemani, R. R. \& Running, S. W., 2002. Global products of vegetation leaf area and fraction absorbed PAR from year one of MODIS data. Remote Sensing of Environment, 83: 214-231. http://dx.doi.org/10.1016/S0034-4257(02)00074-3.

Nemani, R., Pierce, L., Running, S. \& Band, L., 1993. Forest ecosystem processes at the watershed scale: sensitivity to remotely sensed leaf-area index estimates. International Journal of Remote Sensing, 14 (13): 2519-2534 http:// dx.doi.org/10.1080/01431169308904290.

Norman, J. M. \& Campbell, G. S., 1989. Canopy structure, Plant Physiological Ecology: Field Methods and Instrumentation. R. W. Pearcy, et al., 301-326, Chapman and Hall. pp.: 301-326. New York.

Pausas, J. G., 2004. Changes in fire and climate in the eastern Iberian Peninsula (Mediterranean Basin). Climatic Change, 63: 337350. http://dx.doi.org/10.1023/B:CLIM.0000018508.94901.9c.

Pausas, J.G., 2012. Incendios forestales. Catarata y CSIC, 128 pp., Zaragoza.

Pérez-Cabello F., 2002. Paisajes forestales y fuego en el Prepirineo occidental oscense. Un modelo regional de reconstrucción ambiental. Serie Investigación 33. Publicaciones del Consejo de Protección de la Naturaleza de Aragón, 358 pp., Zaragoza.

Pérez-Cabello, F., Cerdà, A., de la Riva, J., Echeverría, M. T., García-Martín, A., Ibarra, P., Lasanta, T., Montorio, R. \& Palacios, V., 2012. Micro-scale post-fire surface cover changes monitored using high spatial resolution photography in a semiarid environment: A useful tool in the study of post-fire soil erosion processes. Journal of Arid Environments, 76: 88-96. http://dx.doi.org/10.1016/j.jaridenv.2011. 08.007 .

Romo Leon, J. R., van Leeuwen, W. J. D. \& Casady, G. M., 2012. Using MODIS-NDVI for the Modeling of Post-Wildfire Vegetation Response as a Function of Environmental Conditions and Pre-Fire Restoration Treatments. Remote Sensing, 4(3): 598-621. http://dx.doi.org/10.3390/rs4030598.

Ruíz de la Torre, J. 1990a. Mapa forestal de España. Escala 1:200.000. Hoja 8-3. Huesca. Ministerio de Agricultura, Pesca y Alimentación. Madrid.

Ruíz de la Torre, J. 1990b. Mapa forestal de España. Escala 1:200.000. Hoja 7-4. Zaragoza. Ministerio de Agricultura, Pesca y Alimentación. Madrid.

Serbin, S. P., Ahl, D. E. \& Gower, S. T., 2013. Spatial and temporal validation of the MODIS LAI and FPAR products across a boreal forest wildfire chronosequence. Remote Sensing of Environment, 133: 71-84. http://dx.doi. org/10.1016/j.rse.2013.01.022.

Steinberg, D. C., Goetz, S. J., \& Hyer, E. J., 2006. Validation of MODIS FPAR products in boreal forests of Alaska. IEEE Transactions on Geoscience and Remote Sensing, 44: 18181828. http://dx.doi.org/10.1109/TGRS.2005.862266.

Tanase, M., de la Riva, R. R., Santoro, M., Pérez-Cabello, F. \& Kasischke, E., 2011. Sensitivity of SAR data to post-fire forest regrowth in Mediterranean and boreal forests. Remote Sensing of Environment, 115: 2075-2085. http://dx.doi. org/10.1016/j.rse.2011.04.009.

Turner, D., Cohen, W., Kennedy, R., Fassnacht, K. \& Briggs, J., 1999. Relationships between leaf area index and Landsat TM spectral vegetation indices across three temperate zone sites. Remote Sensing of Environment, 70: 2-68. http://dx.doi.org/10.1016/S0034-4257(99)00057-7. 
Vicente-Serrano, S. M., Pérez-Cabello, F. \& Lasanta, T., 2011. Pinus halepensis regeneration after a wildfire in a semiarid environment: assessment using multitemporal Landsat images. International Journal of Wildland Fire, 20: 195-208. http://dx.doi.org/10.1071/WF08203.

Viedma, O., Melia, J., Segarra, D. \& García-Haro, J., 1997. Modeling rates of ecosystem recovery after fires by using Landsat TM data. Remote Sensing of Environment, 61: 383-398. http://dx.doi.org/10.1016/S0034-4257(97)00048-5.

Villar, L., 1990. Vegetación. En: Mapa forestal de España. Escala 1:200.000. Hoja 7-4. Zaragoza, 53- 78 pp., ETSIM e ICONA. Madrid.
Yang, W. Z., Tan, B., Huang, D., Rautiainen, M., Shabanov, N. V., Wang, Y., et al., 2006. MODIS leaf area index products: From validation to algorithm improvement. IEEE Transactions on Geoscience and Remote Sensing, 44: 1885-1898. http://dx.doi.org/10.1109/TGRS.2006.871215.

Zarate-Valdez, J. L., Whiting, M. L., Lampinen, B. D., Metcalf, S., Ustin, SL. \& Brown, P.H., 2012. Prediction of leaf area index in almonds by vegetation indexes. Computers and Electronics in Agriculture, 85: 24-32. http://dx.doi. org/10.1016/j.compag.2012.03.009. 